\title{
Non-monotonic Pressure Field induced by Ionic Diffusion in Charged Thin Films
}

DOI:

10.1021/acs.iecr.6b00842

\section{Document Version}

Accepted author manuscript

Link to publication record in Manchester Research Explorer

\section{Citation for published version (APA):}

Joekar-Niasar, V., \& Mahani, H. (2016). Non-monotonic Pressure Field induced by lonic Diffusion in Charged Thin Films. Industrial and Engineering Chemistry Research, 55(21), 6227-6235.

https://doi.org/10.1021/acs.iecr.6b00842

\section{Published in:}

Industrial and Engineering Chemistry Research

\section{Citing this paper}

Please note that where the full-text provided on Manchester Research Explorer is the Author Accepted Manuscript or Proof version this may differ from the final Published version. If citing, it is advised that you check and use the publisher's definitive version.

\section{General rights}

Copyright and moral rights for the publications made accessible in the Research Explorer are retained by the authors and/or other copyright owners and it is a condition of accessing publications that users recognise and abide by the legal requirements associated with these rights.

\section{Takedown policy}

If you believe that this document breaches copyright please refer to the University of Manchester's Takedown Procedures [http://man.ac.uk/04Y6Bo] or contact uml.scholarlycommunications@manchester.ac.uk providing relevant details, so we can investigate your claim.

\section{OPEN ACCESS}


This document is confidential and is proprietary to the American Chemical Society and its authors. Do not copy or disclose without written permission. If you have received this item in error, notify the sender and delete all copies.

\section{Non-monotonic Pressure Field induced by Ionic Diffusion in Charged Thin Films}

\begin{tabular}{|r|l|}
\hline Journal: & Industrial \& Engineering Chemistry Research \\
\hline Manuscript ID & ie-2016-008424.R1 \\
\hline Manuscript Type: & Article \\
\hline Date Submitted by the Author: & n/a \\
\hline Complete List of Authors: & $\begin{array}{l}\text { Joekar-Niasar, Vahid; The University of Manchester, School of Chemical } \\
\text { Engineering and Analytical Science } \\
\text { Mahani, Hassan; Shell, Shell R\&D laboratories, The Netherlands }\end{array}$ \\
\hline
\end{tabular}

SCHOLARONE

Manuscripts 


\title{
Non-monotonic Pressure Field induced by Ionic
}

\section{Diffusion in Charged Thin Films}

\author{
Vahid Joekar-Niasar, ${ }^{* \dagger}$ and Hassan Mahani ${ }^{\ddagger}$ \\ School of Chemical Engineering and Analytical Science, University of Manchester, Manchester, \\ United Kingdom, and Innovation and R\& D, Shell Global Solutions International, Rijswijk, the \\ Netherlands \\ E-mail: vahid.niasar@manchester.ac.uk
}

\begin{abstract}
Dynamics of pressure field evolution inside thin films under the effect of ionic strength gradient is not well understood. Dynamics of the pressure field is important as it controls the film hydrodynamics and also change of contact angle due to the change of ionic strength. The major two potentials building the total pressure in thin films are osmotic and electrostatic potentials. In thin films, these two components are working against each other as while the reduction of ionic strength will decrease the osmotic pressure, it will increase the electrical double layer thickness. However, this interaction is controlled by transport of ions and the transport time-scale. Here, we present a model that couples Nernst-Planck and Poisson equations to simulate ionic transport and also Stokes equation augmented by Maxwell stress tensor (MST) to simulate the pressure field. Results show a highly nonlinear behaviour in the pressure field that is initiated by diffusion of the ions in a channel which is initially filled by a high ionic strength electrolyte and is exposed to a bulk solution with a lower ionic strength.
\end{abstract}

*To whom correspondence should be addressed

${ }^{\dagger}$ School of Chemical Engineering and Analytical Science, University of Manchester, Manchester, United Kingdom

†nnovation and R\& D, Shell Global Solutions International, Rijswijk, the Netherlands 


\begin{abstract}
Results show that diffusion length (transport length) and the overlapping of the double layers affect the pressure field significantly. The results imply that in thin films where ionic diffusion is expected, interfaces can deform due to the nonlinear pressure field, which is triggered by the asymmetric and multi-directional transport of ions. This brings a new insight into thin film hydrodynamics that can contribute to understanding the dimple formation in thin films.
\end{abstract}

\title{
Ionic transport and thin film hydrodynamics
}

Ionic transport in micro/nano channels and thin films have been intensively studied from atomistic and mechanistic point-of-view for different industrial and natural systems. ${ }^{1-4}$ For example, the membrane technology is based on controlling the transport (diffusion) of ions by a selective electric field in nano-pores, which makes the membrane permeable only to counter ions. In particle physics, interaction between colloids and interfaces ${ }^{5,6}$ due to electrostatic forces control the total force and finally attachment and detachment of the particles, which are commonly calculated using the DLVO theory. However, the DLVO analysis cannot provide information about the dynamics of the pressure field. Lamella stability in foams is another important application where understanding of pressure evolution inside the water films and ionic diffusion and their time scale control the foam performance. ${ }^{7}$ Not only the rate of film thinning and thickening is controlled by the surface and electrolyte charge densities but also the shape of interfaces is controlled by the surface forces and film thickness as experimentally observed ${ }^{8,9}$ and hydrodynamically modelled in other studies. ${ }^{10}$ When a bubble approaches another surface, the curvature of the interface in the central point of the lens between bubble and surface will be reversed so that the central part beneath the bubble will have larger film thickness compared to the rim of the lens. This is referred to as dimple. The dimple will disappear when the system reaches a new mechanical equilibrium state. ${ }^{11}$ Dynamics of the pressure field to analyse the dimple formation has been intensively studied from the hydrodynamic perspective. ${ }^{11-16}$ The Reynolds lubrication theory for thin films augmented by an ad hoc approximation of surface forces has been employed to simulate the pressure field. However, this theory is unable to provide any information about the dynamics of electrical field and surface 
forces due to charge migration.

The interaction of ionic diffusion and thin film hydrodynamics can be found in other industrial applications such as petroleum engineering. Wettability alteration by lowering the ionic strength is an emerging technology in petroleum engineering, referred to as "low salinity water flooding" (LSF) that can improve the hydrocarbon extraction efficiency. It is conjectured that LSF modifies the wettability by reducing the ionic strength and increase of disjoining pressure. ${ }^{17}$ Experimental observations ${ }^{17}$ (see Figure 1) support the hypothesis that wettability can be significantly modified by replacing the high ionic strength electrolyte with a low ionic strength electrolyte. As a result, the solid surface becomes less oil-wet and oil may get detached from the surface. This cannot happen if ionic diffusion does not occur in thin films. As shown in Figure 1, this process is slower than simple Fickian diffusion. Churaev ${ }^{18}$ has shown that a contact angle (wettability) is directly linked to the disjoining pressure in a thin film. Thus, to understand the dynamics of contact angle variation, one should understand the dynamics of disjoining pressure in thin films, which is the main objective of this paper. We develop a computational model that integrates ionic transport (Nernst-Planck theory) and electrical field (Poisson theory) with Maxwell Stress Tensor to compute the pressure field in time. To reduce the computational complexity, we assume a rigid domain (no interface deformation). We will show how the overlapping of double layers and ionic diffusion length influence the evolution of pressure.

\section{Theory}

\section{Electrical double layer}

In the vicinity of a charged surface two different layers are conceptualized (Figure 2). The first layer comprises ions adsorbed onto the surface due to chemical interactions and the second layer is composed of ions attracted to the surface charge via the Coulombic forces. The second layer is composed of free ions that can move in the fluid due to any external gradient (such as pressure, electric potential, concentration). Therefore, it is referred to as "diffuse layer". The double layer 

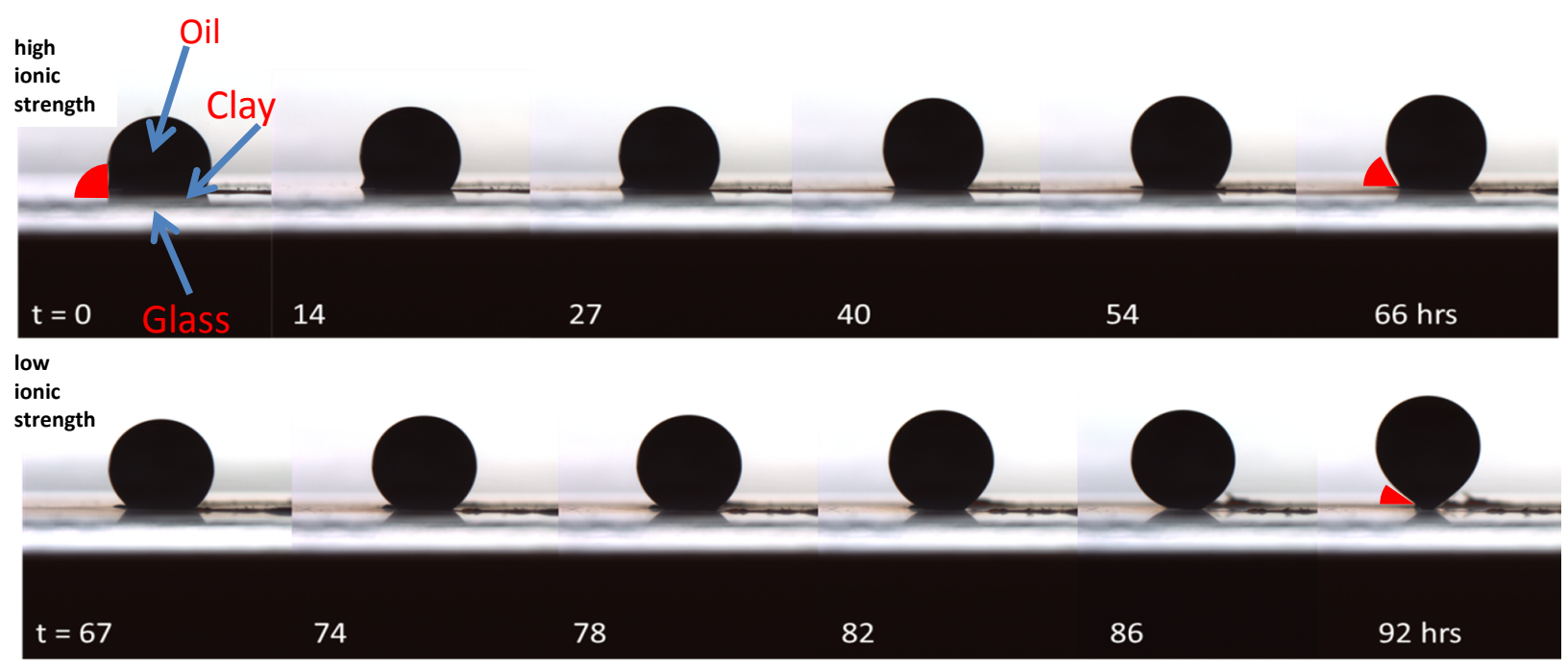

Figure 1: Impact of decreasing the ionic strength of an electrolyte on changing the wettability of a clay-covered glass surface towards more water-wet conditions. Crude oil has affinity to the surface at high ionic strength and by replacing the initial electrolyte with a low ionic strength electrolyte the surface becomes water-wet.

thickness is characterised by the Debye length $\left(\lambda_{D}\right)$.

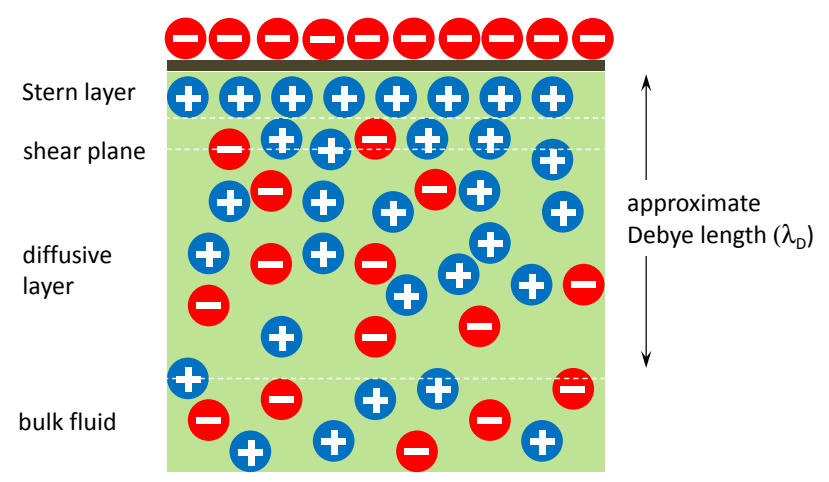

Figure 2: A conceptual presentation of the electrical double layer consisting of the Stern and diffusive layer. The approximate distance from the charge interface to reach a bulk fluid is introduced by Debye length $\left(\lambda_{D}\right)$. Zeta potential is theoretically the electric potential at the shear plane.

\section{Electric field}

Since the objective is to develop a numerical model to estimate the change in disjoining pressure due to the change of electrostatic forces with time, it is needed to simulate the coupled electric field using the Poisson equation and transport of the ions using the Nernst-Planck equation. The coupled 
Poisson-Nernst-Planck (PNP) model is briefly explained here. Under non-equilibrium conditions, the Poisson equation relates the electric potential $(\psi)$ to the net charge density:

$$
\nabla^{2} \psi=-\frac{\rho_{e}}{\varepsilon}
$$

where, $\varepsilon$ is the permittivity of the medium, $\rho_{e}$ is the net charge density as the difference between cations and anions: $\rho_{e}=\sum e z_{i} n_{a} c_{i}$, where $z_{i}$ and $n_{a}$ denote the valance of the ion $i$ and Avogadro number, respectively. Also, $e$ denotes the charge of a proton, and $c_{i}$ denotes the mol concentration of the ion $i$. Under equilibrium conditions, the electric potential would decay to zero at a distance $\lambda_{D}$ from the charged surface. This distance, referred to as the Debye length, is inversely proportional to the square root of the ionic strength, $\Gamma$, written as:

$$
\begin{aligned}
& \lambda_{D}=\left(\frac{\varepsilon k_{B} T}{2 e^{2} \Gamma}\right)^{0.5} \\
& \Gamma=0.5 \sum_{i} c_{b} n_{a} z_{i}^{2}
\end{aligned}
$$

$k_{B}$ is the Boltzmann constant and $T$ is the temperature and $c_{b}$ is the bulk concentration where $\psi$ is zero (electro-neutral condition). The spatial distribution of ions is given by the Boltzmann distribution, as follows:

$$
c_{i}=c_{b} \exp \left(-\frac{z_{i} e \psi}{k_{B} T}\right)
$$

\section{Transport of ions}

Based on the Nernst-Planck relation, the mass balance of the non-reactive ion $i$ is given:

$$
\begin{gathered}
\partial_{t} c_{i}+\nabla \cdot \mathbf{j}_{i}=0 \\
\mathbf{j}_{i}=c_{i} \mathbf{v}-\frac{D_{i}}{k_{B} T} c_{i} \nabla \mu_{i} \\
\mu_{i}=n_{a}\left(e z_{i} \psi+k_{B} T \ln c_{i}\right)
\end{gathered}
$$


This equation includes advection, dispersion and electro-migration of ions, in which, $D_{i}$ denotes the binary diffusion coefficient for ion $i$, and $\mu_{i}$ denotes the electro-chemical potential of ions. Note that in our study, the advective part is negligible $(\mathbf{v}=0)$.

\section{Total force acting on the surface}

One common approach to calculate the force acting on the surface is to use the Maxwell Stress Tensor (MST). The electrical origin of force acting on the surface requires the exact solution of the electric field and integration of the MST over the surface. Including osmotic pressure (П), the Maxwell stress tensor can be written as follows: ${ }^{19}$

$$
T_{i j}=\Pi \delta_{i j}+0.5 \varepsilon|\mathbf{E}|^{2} \delta_{i j}-\varepsilon E_{i} E_{j}
$$

where $\delta_{i j}$ is the Kronecker delta and $\mathrm{i}, \mathrm{j}=\mathrm{x}, \mathrm{y}, \mathrm{z}$.

Most studies dealing with the calculation of pressure inside a thin film are restricted to equilibrium conditions. At equilibrium Poisson-Boltzmann equation is valid and is used to relate the osmotic pressure in the double layer to the bulk osmotic pressure. This gives the differential osmotic pressure as. ${ }^{20}$

$$
\Pi_{d}=n_{a} k_{B} T\left(c^{+}+c^{-}-2 c_{b}\right)
$$

This does not hold under non-equilibrium conditions as $c^{ \pm} \neq c_{b}^{ \pm} \exp (\mp \psi)$. To calculate the relative pressure difference a virtual bulk concentration has been locally defined as $c_{b}^{ \pm}=c^{ \pm} \exp ( \pm \psi)$. This leads to a the differential osmotic pressure appropriate to calculate the relative pressure:

$$
\Pi_{d}=n_{a} k_{B} T \Sigma c^{ \pm}(1-\exp ( \pm \psi))
$$

\section{Pressure evolution inside the channel}

To calculate the pressure field inside the film or channel, Navier-Stokes equation augmented with the body force due to the electrochemical potential of the system is used. ${ }^{21,22}$ The electrostatics 
and hydrodynamics are coupled together through the Maxwell stress tensor based on the following relation:

$$
\rho \frac{D \mathbf{u}}{D t}=-\nabla p+\mu \nabla^{2} \mathbf{u}+\nabla \cdot \mathbf{T}
$$

where $\mu$ is fluid dynamic viscosity, $p$ is pressure and $\rho$ is fluid density. This is the full form of the conservation of the momentum in thin films.

The other simpler alternative for simulating hydrodynamics of thin (charged or neutral) films is the application of "Reynolds lubrication theory". In thin film applications, where the fluid motion is in the Stokes regime, the Reynolds lubrication theory has been used where film thickness and film pressure as a function of time and position in radial form $h(r, t), p(r, t)$ have been modelled. ${ }^{23-26}$ For example, Webber et al. ${ }^{23}$ employed the Reynolds lubrication theory to study dimple formation. They explained that dimple arises from the combined effects of decrease of hydrodynamic pressure and monotonic repulsive double layer interactions. Joye et al. ${ }^{27}$ measured the rate of film thinning in a symmetrical foam film and simulated it by including the disjoining pressure in the film theory using lubrication approximation. The disjoining pressure is proposed only as a function of film thickness. They concluded that with inclusion of the disjoining pressure, the film drains relatively fast compared to a thick uniform equilibrium film. In the opposite way, inclusion of the droplet deformation had a reasonable agreement with the experiments. In none of these studies using the lubrication theory, the impact of transport of ions on disjoining pressure has been simulated. Also the time evolution of disjoining pressure has not been studied. Therefore, the Navier-Stokes formulation of disjoining pressure is preferred as it can be coupled with the transport of ions.

Since our study system is under the diffusion condition, the advective velocity field can be ignored that reads:

$$
\nabla p=\nabla \cdot \mathbf{T}
$$




\section{Numerical modelling}

\section{Cylindrical form of the governing equations}

Since the thin electrolyte film squeezed between the oil droplet and the surface can be idealized as a disc with axi-symmetric properties (inspired by the experiment shown in Figure 1), the equations can be rewritten in the cylindrical coordinates. Note that in this modelling exercise, the domain is rigid while in the experiment the oil interface in contact with the surface is deformed as the more water-wet status is attained. The size of the rigid domain based on the dimensionless parameters is defined as $-h / \lambda_{D_{\mathrm{H}}}<\bar{z}<h / \lambda_{D_{\mathrm{H}}}$ and $-R / \lambda_{D_{\mathrm{H}}}<\bar{r}<R / \lambda_{D_{\mathrm{H}}}$ as shown in Figure 3. $\lambda_{D_{\mathrm{H}}}$ is the Debye length in high ionic strength (initial condition in this study) for any composition of the fluid. Due to the axi-symmetric condition we will have primary variables as $c_{i}=c_{i}(z, r)$ and $\psi=\psi(z, r)$. We define other dimensionless parameters as follows: $\bar{c}_{i}=c_{i} / c_{b}^{H}, \lambda_{D_{\mathrm{H}}}=\left(\frac{\varepsilon k_{B} T}{e^{2} n_{a} \sum z_{i}^{2} c_{b}^{H}}\right)^{1 / 2}, \bar{r}=r / \lambda_{D_{\mathrm{H}}}$, $\bar{z}=z / \lambda_{D_{\mathrm{H}}}, \bar{\psi}=\frac{e \psi}{k_{B} T}, \kappa=h / \lambda_{D_{\mathrm{H}}}, G=R / h, t_{D}=R^{2} / D_{\text {ref }}, \bar{t}=t / t_{D}$. We can select one of the diffusion coefficients as the reference diffusion coefficient $D_{\text {ref }}$. Based on the aforementioned parameters the dimensionless form of the Poisson-Boltzmann equations gives:

$$
\partial_{\bar{z}}\left(\partial_{\bar{z}} \bar{\psi}\right)+\frac{1}{\bar{r}} \partial_{\bar{r}}\left(\bar{r} \partial_{\bar{r}} \bar{\psi}\right)=\sinh \bar{\psi}
$$

The equilibrium initial conditions is defined by equation 13 , but then under dynamic conditions the Poisson equation is applied, as follows:

$$
\partial_{\bar{z}}\left(\partial_{\bar{z}} \bar{\psi}\right)+\frac{1}{\bar{r}} \partial_{\bar{r}}\left(\bar{r} \partial_{\bar{r}} \bar{\psi}\right)=-0.5 \sum z_{i} \bar{c}_{i}
$$

For the transport, the dimensionless Nernst-Planck would be given as :

$$
\partial_{\bar{t}} \bar{c}_{i}=\omega_{i} G^{2} \kappa^{2}\left[\partial_{\bar{z}}\left(\partial_{\bar{z}} \bar{c}_{i}\right)+\frac{1}{\bar{r}} \partial_{\bar{r}}\left(\bar{r} \partial_{\bar{r}} \bar{c}_{i}\right)+z_{i} \partial_{\bar{z}}\left(\bar{c}_{i} \partial_{\bar{z}} \bar{\psi}\right)+\frac{z_{i}}{\bar{r}} \partial_{\bar{r}}\left(\bar{c}_{i} \bar{r} \partial_{\bar{r}} \bar{\psi}\right)\right]
$$


a)

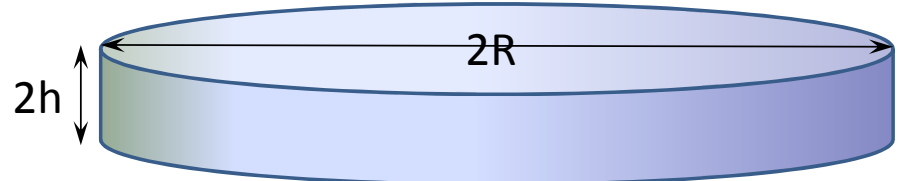

b)

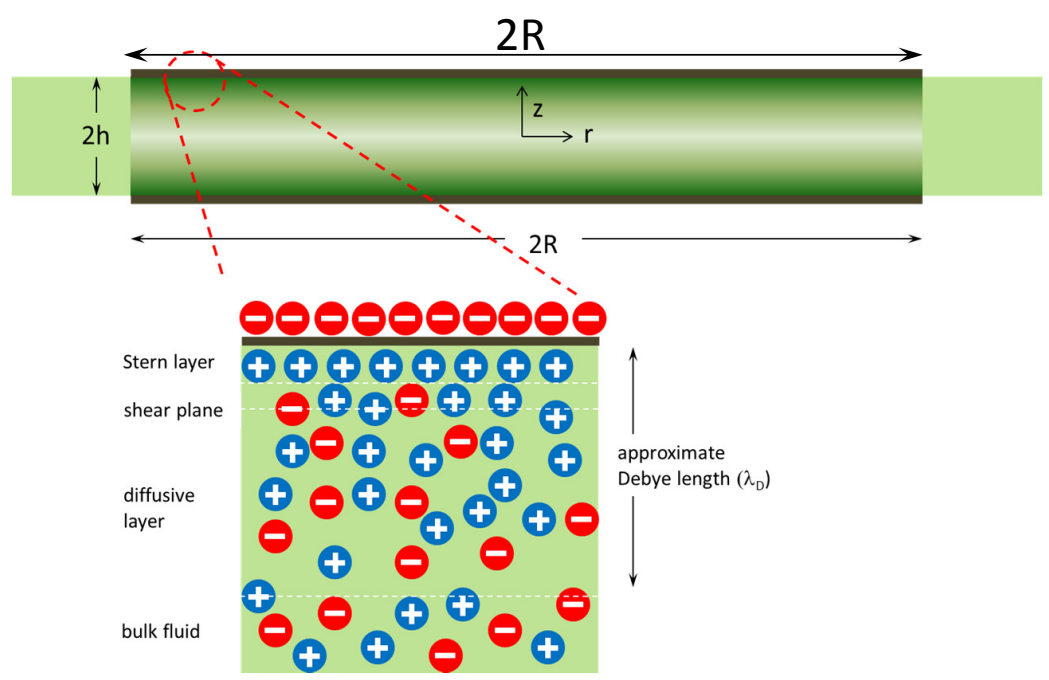

Figure 3: a) The very thin film squeezed between the oil droplet and the surface is idealized as a disc with a height of $2 h$ and a diameter of $2 R$. Surfaces are assumed to be flat. b) A cross section of the simulated cylindrical domain with a diameter of $2 R$ and a height of $2 h$. The cylinder is initially filled with an electrolyte with a higher ionic strength. The top and bottom boundaries are the charged interfaces and the right and left boundaries are open to the electrolyte at a constant lower ionic strength. 
Since the binary diffusion coefficients for the ions are not necessarily the same, $\omega_{i}$ has been introduced as follows, $\omega_{i}=D_{i} / D_{\text {ref }}$.

Taking the divergence from both sides of Equation 12 will result the following dimensionless equation in the cylindrical form.

$$
\partial_{\bar{z}}\left(\partial_{\bar{z}} \bar{p}\right)+\frac{1}{\bar{r}} \partial_{\bar{r}}\left(\bar{r} \partial_{\bar{r}} \bar{p}\right)=\partial_{\bar{z}}\left(\partial_{\bar{z}} \bar{\Pi}\right)+\frac{1}{\bar{r}} \partial_{\bar{r}}\left(\bar{r} \partial_{\bar{r}} \bar{\Pi}\right)+\partial_{\bar{z}}\left(\bar{\rho}_{e} \partial_{\bar{z}} \bar{\psi}\right)+\frac{1}{\bar{r}} \partial_{\bar{r}}\left(\bar{r} \bar{\rho}_{e} \partial_{\bar{r}} \bar{\psi}\right)
$$

where $\bar{p}=\frac{p}{n_{a} k_{B} T}$ and $\bar{\Pi}=\frac{\Pi_{d}}{n_{a} k_{B} T}$.

\section{Boundary and initial conditions}

For modelling solid fluid interactions, the electrostatic interface condition is extremely important and can control the transport of ions as well as evolution of the disjoining pressure. There are two major boundary conditions for charged surfaces; constant charge density and constant potential conditions. Based on the discussion provided in the Supporting Information, we propose that the constant charge density condition can represent the physical conditions more satisfactorily compared to the constant potential conditions. We assume a constant charge density on top and bottom boundaries of the disc shown in Figure 3. Following the Gauss' law, the charge density $\sigma$ would be related to the gradient of the electric field normal to the surface. ${ }^{28}$ This is a natural boundary condition where the charge density is an intrinsic property of the medium.

$$
\nabla \psi \cdot \mathbf{n}=-\frac{\sigma}{\varepsilon}
$$

By introducing the characteristic charge density, $\sigma_{0}=\frac{\varepsilon k_{B} T}{\lambda_{D_{H}} e}$ and $\bar{\sigma}=\sigma / \sigma_{0}$, the dimensionless form of the boundary condition would be as follows:

$$
\left(\partial_{\bar{z}} \bar{\psi}\right) \cdot n_{z}=-\bar{\sigma}
$$

The side boundaries of the disc shown in Figure 3 are exposed to the bulk fluid. Due to the 
symmetry of the disc, a 2D cross section of the half disc is simulated as shown in Figure 4. The right boundary is exposed to the bulk fluid and the left boundary which is the centre of the disc, $\nabla \psi \cdot \mathbf{n}=0$ or in the dimensionless form $\left(\partial_{\bar{x}} \bar{\psi}\right) \cdot n_{x}=0$.

As explained the right boundary is exposed to the bulk electrolyte at a lower concentration $(0.1)$ and the left boundary, centre of the disc, is at the zero gradient (no flux) of concentration. Zero gradient of concentration is also applied to the top and bottom boundaries.

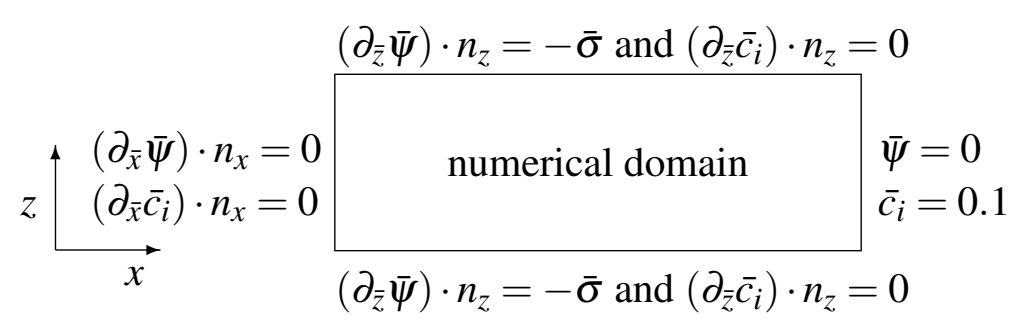

Figure 4: Boundary conditions of electric potential and ionic concentration. Top and bottom boundaries are at constant charge density for the electric potential and no flux condition for the concentration. The right boundary, exposed to the bulk fluid, is at zero electric potential and dimensionless bulk concentration of 0.1 . The left boundary - being the axis of the cylindrical domain - is at no flux boundary condition for both electric potential and concentration.

The initial condition is dictated by the equilibrium Poisson-Boltzmann equation, given by Equation 13 under high ionic strength conditions. To solve the initial equilibrium state, a nonlinear system of equations (Equation 13) should be solved. We used the HSL-MA57 package from HSL numerical library (The HSL Mathematical Software Library ${ }^{29}$ ) which employs the multifrontal method to solve sparse symmetric systems. This is a direct approach based on a sparse variant of Gaussian elimination. The nonlinear Poisson-Boltzmann solver has been tested for a 1D case, which is in excellent agreement with the analytical solution. Details can be found in the Supporting Information.

For transient conditions, the Poisson equation (Equation 14) should be solved. A diagonally scaled biconjugate gradient sparse $A x=b$ solver from SLATEC numerical library ${ }^{30}$ is used. The 
grid resolution is chosen to be 0.01 (scaled by the Debye length thickness) in all simulations.

\section{Results and discussion}

\section{Effect of overlapping double layers on ionic diffusion}

The simulations start with an equilibrium condition at high ionic strength (1.0). Exposing the domain to a lower ionic strength (0.1) initiates the diffusion of the ions. Figures 5 (a) and (b) show the concentration maps for cations at the start and end of the simulation. Respectively, Figures 5 (c) and (d) show the concentration maps for anions at the start and end of the simulation. The right boundary of the main is exposed to the bulk concentration (0.1) and the concentration fields develop by getting further into the channel from right to left. To show the dynamics of the ionic

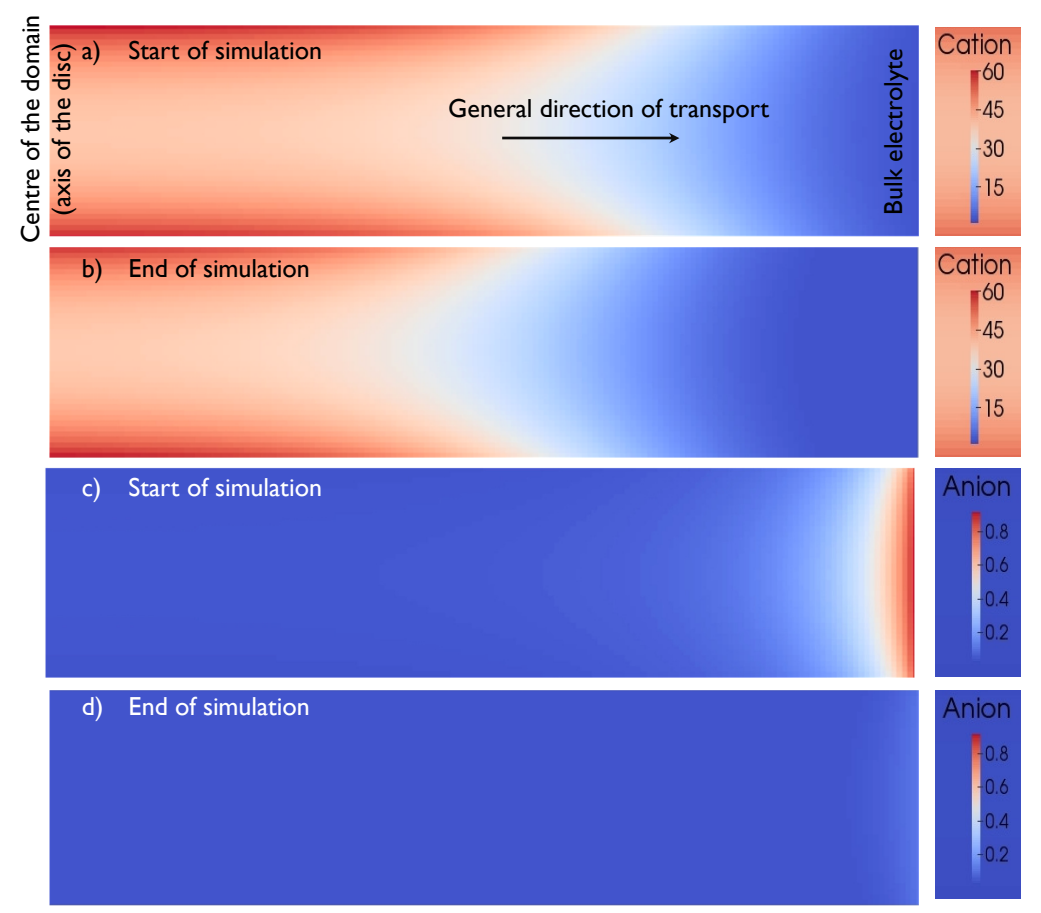

Figure 5: Concentration profiles of cations $(a, b)$ and anions $(c, d)$ at the start and end of one simulation with half of thickness of $\bar{h}=0.2$ and radius of $\bar{R}=2$. Start and end of the simulations are both at equilibrium.

diffusion and electric field evolution, the value of concentration of ions averaged over the cross 
section along the channel have been plotted in Figures 6 (a) and (b) for two simulations $\bar{h}=0.2$, $\bar{R}=2$ and $\bar{h}=1.0$ and $\bar{R}=10$. The red curves show the initial condition and the blue curve show the ultimate equilibrium condition. The dashed curves correspond to cations and the solid curves correspond to anions. Zero in $\mathrm{x}$-axis denotes the centre of the disc. Getting closer to the bulk fluid boundary (the right side of the curves) the difference between the ion concentrations becomes smaller as the electric field gets weaker. Eventually at the boundary the concentrations of ions match the boundary value. The grey curves show the transient conditions of concentrations. As obviously it can been seen, the cations have much slower outward diffusion rate compared to anions, although the absolute valance of the ions and their diffusion coefficients are the same. As a result of ionic diffusion, the electric field enhances the co-ions diffusion. By increasing the channel height (reducing the overlapping double layers), the cations diffusion rate becomes faster and the opposite occurs for anions. In charged systems where the electric field is strong, the diffusion of ions is asymmetric and the symmetry becomes stronger by increase of channel height or reduction of electric field. In our negatively charged system, the effective diffusion coefficient for cations under overlapping conditions, is orders of magnitude smaller than the bulk diffusion coefficient and for anions it is orders of magnitude larger, which is consistent with the results of Mahani et al. 17 Note that due to the negatively charged boundary conditions (similar to natural sandstone rocks covered by clays) the outward ionic diffusion of the cation is more important.

\section{Evolution of pressure in a thin channel}

To analyze the pressure evolution inside the channel, we are interested in estimating the differential pressure, which is the difference between total pressure and osmotic pressure. However, osmotic pressure can be shown by two definitions, transient osmotic pressure (Equation 10) and steady-state osmotic pressure (Equation 9).

In formalism (a) the pressure is calculated based on the definition of local transient osmotic pressure (Equation 10). This approach has a clear advantage as it clearly shows the gradual evolution of the pressure triggered by the reduction of bulk concentration at the boundary and this 

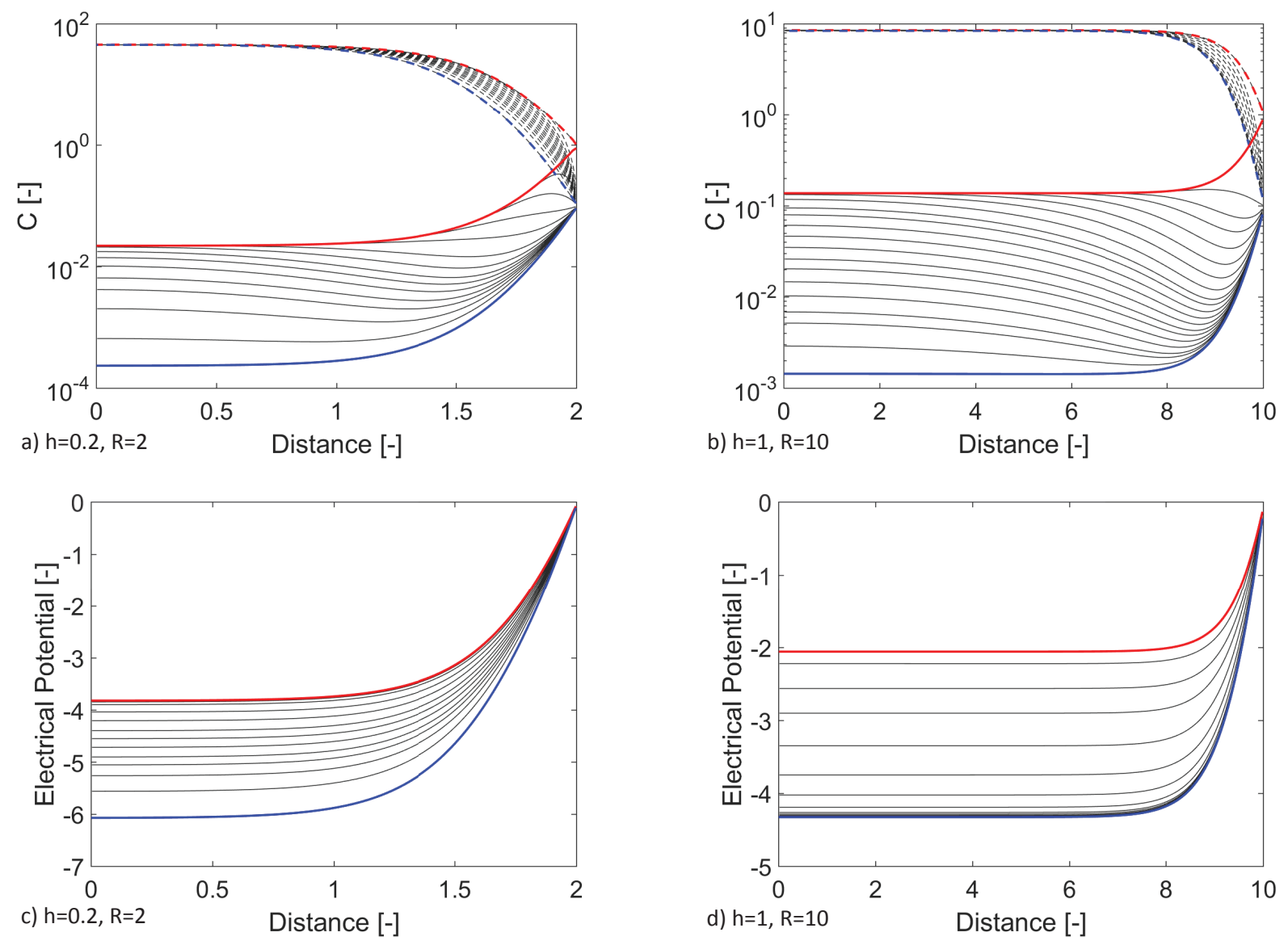

Figure 6: Average concentration profiles for cations (dashed lines) and anions(solid lines) along the channel with a) $\bar{h}=0.2$ and $\bar{R}=2$ and b) $\bar{h}=1.0$ and $\bar{R}=10$. Electric potential evolution along the channel for these two cases have been shown respectively in Figures (c) and (d). Note that red curves show the initial condition (higher ionic strength) and blue curves show the final stage (lower ionic strength) both at equilibrium conditions. 
definition shows how far the system is from the equilibrium status. When the system is at equilibrium, the total pressure at double layer will be equal to the osmotic pressure of the bulk fluid at the boundary.

In formalism (b), the pressure is calculated using the steady-state definition of the osmotic pressure (Equation 9). Since the osmotic pressure at low ionic strength is smaller than the osmotic pressure at high ionic strength, the ultimate equilibrium pressure will be smaller than the initial pressure.

For a system at equilibrium at high ionic strength, the pressure everywhere is the same, resulting in a zero net electostatic pressure based on formalism (a)(red curves in Figures 7)a and c. Then the bulk concentration at the boundary decreases one order of magnitude causing the evolution of electric field and ionic diffusion. After reaching the equilibrium condition at low ionic strength, again the pressure would be the same everywhere equal to the boundary pressure (blue curves in Figures $7 \mathrm{a}$ and c). However based on the formalism (b) by changing the boundary value from high to low concentration, compared to the ultimate equilibrium condition at low ionic strength, the relative pressure would be higher (red dashed curves in Figures $7 \mathrm{c}$ and d). This relative pressure would deteriorate by time when the equilibrium condition is reached (blue curves in Figures $7 \mathrm{c}$ and d).

As Figure 7 (a) shows there will be a pressure build-up inside the domain due to the strengthening of the electric field. This pressure build-up continues till reaching a maximum and afterwards with relaxation of ionic transport with electric field developments, the final equilibrium condition under lower ionic strength is attained (blue curve). As expected the pressure inside the disc should be in balance with the boundary pressure under equilibrium conditions either at start or end of simulations. Interestingly not only the differential pressure build-up inside the disc is non-monotonic, but also along the channel at each time step a non-monotonic behaviour is observed. This nonmonotonic profile of the pressure is very significant at the stage of pressure field relaxation (thick black curve). Following the formalism (a), Figure 7c shows a totally different behaviour where relative negative pressure is developed. Note that this pressure is not an absolute value. For $h=1.0$, 

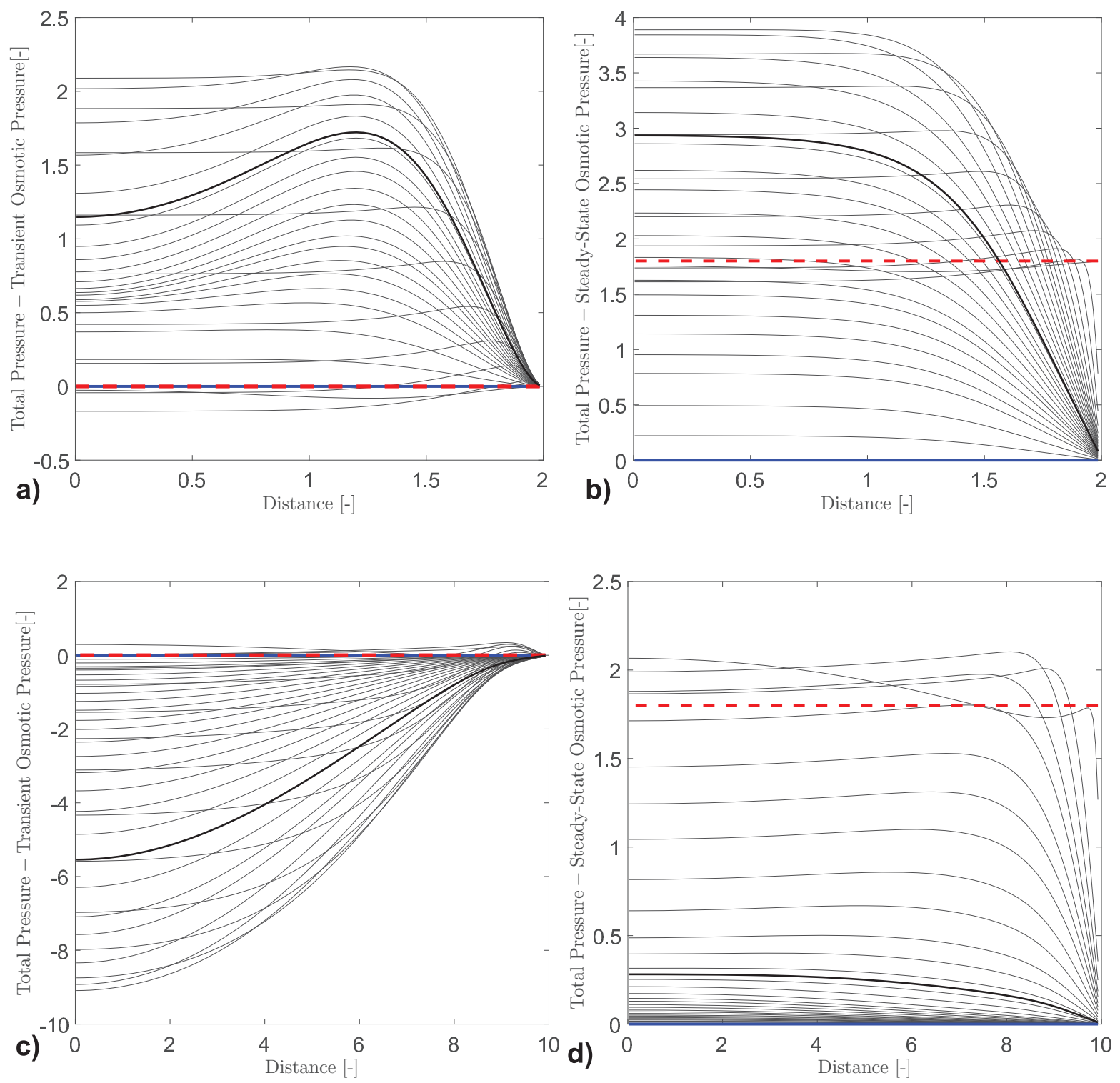

Figure 7: Time evolution of differential pressure relative to the transient osmotic pressure using Equation 10 for a) $\bar{h}=0.2$ and $\bar{R}=2$ and c) $\bar{h}=1.0$ and $\bar{R}=10$. Time evolution of differential pressure relative to the steady-state osmotic pressure using Equation 9 for $\mathrm{b}) \bar{h}=0.2$ and $\bar{R}=2$, and d) $\bar{h}=1.0$ and $\bar{R}=10$. Red dashed line and blue solid line show the initial and final pressure profiles and then intermediate curves have been shown in grey color. Thick black curve shows an intermediate stage just as a guidance. 
the pressure induced by electric field is weaker than the osmotic pressure and due to the easier transport of ions compared to Figure 7a the system reaches equilibrium faster and the electric potential-induced pressure cannot compete with the osmotic-induced pressure. Thus a negative differential pressure is developed. Similar to Figure 7a, at the end of simulation an equilibrium condition is achieved where relative pressure is equal to zero everywhere.

In formalism (b) by reducing the bulk concentration by one order of magnitude, there will be a higher pressure all over the channel, shown by red curves in Figures $7 \mathrm{~b}$ and $\mathrm{d}$ for two different overlapping conditions $\bar{h}=0.2, \bar{R}=1.0$ and $\bar{h}=1.0, \bar{R}=5.0$. Figure $7 \mathrm{~b}$ clearly shows a nonmonotonic pressure change where there will be a gradual increase of pressure and then the pressure field will be relaxed reaching to the equilibrium condition. This is not the case for $h=1.0$ where the monotonic reduction of the pressure inside the channel can be seen. As clearly shown here the overlapping conditions play a significant role in the pressure field evolution.

This non-monotonic pressure field in thin films/channels has been also addressed in dimple formation problem. For example, Webber et al. ${ }^{23}$ measured the dynamics of surface forces between a deformable tetradecane oil drop anchored on the cantilever of Atomic Force Microscope (AFM) and similar oil drops on the substrate. They employed the Reynolds lubrication theory to estimate the hydrodynamics of thin films. They explained that dimple (which indicates a non-monotonic pressure field) arises from the combined effects of decrease of hydrodynamic pressure and monotonic repulsive double layer interactions. Also Goodall et al. ${ }^{31}$ measured the film thickness between an oil droplet and a solid surface while the oil droplet was approaching towards the surface. They observed that initially a dimple is formed and in time this dimple will be drained. Ultimately a flat surface will be obtained. They observed that by film thinning and double layer overlapping, the drainage rate slows down. They discussed that effects of electrolyte concentration on hydrodynamic factors (such as interfacial tension) were negligible in their study. Thus, variation of the film drainage were not due to the hydrodynamic factors, and are sensitive to the electrolyte concentrations mainly due to double layer overlapping. These findings although based on deformable interfaces support our results where the nonlinear asymmetric multi-directional transport of ions 
induce a nonlinear pressure field that can initiate interface deformation.

Also the importance of the diffusion length for the non-monotonic pressure field has been echoed in other studies ${ }^{32}$ where authors concluded that dimple formation is happening in films under hydrophobic conditions when the planar radius of the film under the droplet is much larger than the film thickness. As they did not observe dimple formation in smaller diffusion length.

\section{Contribution of pressure dynamics to wettability change in natural rock- crude oil-brine systems}

The simulation results under dynamic conditions clearly show the effect of electric potential field and film thickness on the behavior of ionic diffusion for a given charged system. This was shown for the cases where ionic strength of the surrounding is reduced compared to the resident high ionic strength inside the film. This change disturbs the initially established thermodynamic equilibrium condition of the system. Therefore diffusion of ions kicks in between the film and the surrounding environment. The diffusion process at thinner films is more limited and significantly reduced due to the electric field (since a field of potential gradient is imposed on concentration gradient), which is found to result in a far slower than the pure Fickian diffusion. This is because the mobility of ions is strongly affected by the presence of electric charges. This was severe for the ions having the opposite charge as the interfaces. In this system, the dynamics of pressure is characterized by first a transient pressure build-up in the film followed by slight relaxation of the pressure as the ions concentration reaches equilibrium concentration. As the film thickness decreases, the contribution of osmotic pressure is reduced compared to electro-static, which implies that osmotic pressure contribution become negligible in very thin films. Under the (overall) increase of disjoining pressure due to reduction of ionic strength, the system in over-balanced (i.e. increase of repulsion force by expansion of electrical double layers) which can lead to expansion of the film and deformation of fluid-fluid interface and eventually movement of the interfaces apart, in case the film is not geometrically constrained. The results of study in this paper is very relevant for a number of applications in Improved and Enhanced Oil Recovery where the wettability state 
of reservoir affects significantly the oil recovery. An important determining factor of wettability is the water film thickness. Typically in water-wet rocks, the film thickness is large (can exceed 10-20nm). In oil-wet systems the film thickness can be relatively much thinner. A more specific example is rock, oil, brine system where oil can be the wetting fluid under high ionic strength conditions. Oil can directly bind to the rock by non-DLVO forces (such as acid-base interactions) or can indirectly bind through a water-film (of thickness 1-10nm) separating oil and rock interfaces. Due to the polar components in crude oil and existence of clays particle on surface of sandstone rocks, both oil and rock are highly charged (in case of sandstone rocks, both are negatively charged based on negative zeta potential measurements ${ }^{17}$ ). The wettability can be then modified to more water-wetting be reduction of ionic strength, which can be explained by diffusion of the low ionic strength water to the contact line/film. Under low ionic strength conditions the double-layers become over-lapping which leads to expansion of film and detachment of oil from mineral surface. It is noteworthy that in the previous work, ${ }^{17}$ it was found that the wettability alteration by reduction of ionic strength was unexpectedly slow (time scale was 10-20 times slower than pure diffusion process). This was hypothesized to be related to slow ionic diffusion process owing to the fact that the transport of ions in the film is significantly affected by the electric charges or potential around oil and rock interfaces and the second term in the Nernst-Planck equation becomes dominant. The dynamic simulation results of this study are very consistent with this and can provide a plausible explanation. Nevertheless, the diffusion process can be affected by several other factors such as surface roughness of rock, non-uniform film thickness and charge heterogeneity of the interfaces. These factors were not in the scope of this work to study, however it is certainly worth investigating to answer to what extend wettability alteration can be affected by these factors.

\section{References}

(1) Hu, H.; Weinberger, C. R.; Sun, Y. Model of Meniscus Shape and Disjoining Pressure of Thin Liquid Films on Nanostructured Surfaces with Electrostatic Interactions. The Journal of Physical Chemistry C 2015, 119, 11777-11785. 
(2) El-Kady, M. F.; Strong, V.; Dubin, S.; Kaner, R. B. Laser Scribing of High-Performance and Flexible Graphene-Based Electrochemical Capacitors. Science 2012, 335, 1326-1330.

(3) Wasan, D.; Nikolov, A.; Kondiparty, K. The wetting and spreading of nanofluids on solids: Role of the structural disjoining pressure. Curr. Opin. Colloid Interface Sci. 2011, 16, 344 349.

(4) Yaroshchuk, A.; Bruening, M. L.; Bernal, E. E. L. Solution-Diffusion-Electro-Migration model and its uses for analysis of nanofiltration, pressure-retarded osmosis and forward osmosis in multi-ionic solutions. J. Membr. Sci. 2013, 447, 463 - 476.

(5) Trefalt, G.; Behrens, S. H.; Borkovec, M. Charge Regulation in the Electrical Double Layer: Ion Adsorption and Surface Interactions. Langmuir 2016, 32, 380-400.

(6) Mikelonis, A.; Youn, S.; Lawler, D. DLVO Approximation Methods for Predicting the Attachment of Silver Nanoparticles to Ceramic Membranes. Langmuir 2016, 32, 1723-1731.

(7) Farajzadeh, R.; Krastev, R.; Zitha, P. L. Foam film permeability: Theory and experiment. Adv. Colloid Interface Sci. 2008, 137, 27 - 44.

(8) Derjaguin, B.; Kussakov, M. Anomalous properties of thin polymolecular films V. An experimental investigation of polymolecular solvate (adsorbed) films as applied to the development of a mathematical theory of the stability of colloids. Acta Physicochim. URSS 1939, 10, 2544.

(9) Derjaguin, B.; Kussakov, M. An experimental investigation of polymolecular solvate (adsorbed) films as applied to the development of a mathematical theory of the stability of colloids. Prog. Surf. Sci. 1992, 40, $26-45$.

(10) Frankel, S. P.; Mysels, K. J. On the "dimpling" during the approach of two interfaces. J. Phys. Chem. 1962, 66, 190-191. 
(11) Platikanov, D. Experimental Investigation on the "Dimpling" of Thin Liquid Films. J. Phys. Chem. 1964, 68, 3619-3624.

(12) Hartland, S. The profile of the draining film between a rigid sphere and a deformable fluidliquid interface. Chem. Eng. Sci. 1969, 24, 987 - 995.

(13) Burrill, K.; Woods, D. Change in interface and film shapes for a deformable drop at a deformable liquid-liquid interface: Part I. Film hydrodynamic pressure distribution and interface shapes. J. Colloid Interface Sci. 1969, 30, 511 - 524.

(14) Chevaillier, J. P.; Klaseboer, E.; Masbernat, O.; Gourdon, C. Effect of mass transfer on the film drainage between colliding drops. J. Colloid Interface Sci. 2006, 299, 472-85.

(15) Burrill, K.; Woods, D. Film shapes for deformable drops at liquid-liquid interfaces. II. The mechanisms of film drainage. J. Colloid Interface Sci. 1973, 42, 15 - 34.

(16) Chen, J.-D. Effects of London-van der Waals and electric double layer forces on the thinning of a dimpled film between a small drop or bubble and a horizontal solid plane. J. Colloid Interface Sci. 1984, 98, 329-341.

(17) Mahani, H.; Berg, S.; Ilic, D.; Bartels, W.-B.; Joekar-Niasar, V. Kinetics of Low-SalinityFlooding Effect. SPE Journal 2015, 20, 8-20.

(18) Churaev, N. V. Wetting films and wetting. Revue Phys. Appl. 1988, 23, 975-987.

(19) Stratton, J. A. Electromagnetic Theory; McGraw-Hill, 1941; p 640.

(20) Hans-Jurgen Butt, M. K., Karlheinz Graf Physics and Chemistry of Interfaces, 3rd ed.; Wiley, $2013 ; \mathrm{p} 495$.

(21) Hunter, R. Foundations of Colloid Science; Clarendon Press, Oxford, 1989; Vol. 2.

(22) Revil, A.; Linde, N. Chemico-electromechanical coupling in microporous media. J. Colloid Interface Sci. 2006, 302, $682-694$. 
(23) Webber, G. B.; Edwards, S. A.; Stevens, G. W.; Grieser, F.; Dagastine, R. R.; Chan, D. Y. C. Measurements of dynamic forces between drops with the AFM: novel considerations in comparisons between experiment and theory. Soft Matter 2008, 4, 1270-1278.

(24) Manica, R.; Connor, J. N.; Carnie, S. L.; Horn, R. G.; Chan, D. Y. C. Dynamics of Interactions Involving Deformable Drops: Hydrodynamic Dimpling under Attractive and Repulsive Electrical Double Layer Interactions. Langmuir 2007, 23, 626-637.

(25) Manica, R.; Connor, J. N.; Clasohm, L. Y.; Carnie, S. L.; Horn, R. G.; Chan, D. Y. C. Transient Responses of a Wetting Film to Mechanical and Electrical Perturbations. Langmuir 2008, 24, 1381-1390.

(26) Manica, R.; Chan, D. Y. C. Drainage of the air-water-quartz film: experiments and theory. Phys. Chem. Chem. Phys. 2011, 13, 1434-1439.

(27) Joye, J. L.; Hirasaki, G. J.; Miller, C. A. Dimple formation and behavior during axisymmetrical foam film drainage. Langmuir 1992, 8, 3083-3092.

(28) Israelachvili, J. Intermolecular and Surface Forces, Third Edition; Academic Press, 2010.

(29) HSL. A collection of Fortran codes for large-scale scientific computation. 2014; http:// www.cse.clrc.ac.uk/nag/hsl/.

(30) Fong, K. W.; Jefferson, T. H.; Suyehiro, T.; Walton, L. Guide to the SLATEC common mathematical library. 1993; http://www. netlib.org/slatec.

(31) Goodall, D. G.; Gee, M. L.; Stevens, G. W. An Imaging Reflectometry Study of the Effect of Electrolyte on the Drainage and Profile of an Aqueous Film between an Oil Droplet and a Hydrophilic Silica Surface. Langmuir 2002, 18, 4729-4735.

(32) Pan, L.; Jung, S.; Yoon, R.-H. A fundamental study on the role of collector in the kinetics of bubble-particle interaction. Int. J. Miner. Process. 2012, 106-109, 37 - 41. 


\section{Graphical TOC Entry}

5

1

2

3

6

7

8

9

10

11

12

13

14

15

16

17

18

19

20

21

22

23

24

25

26

27

28

29

30

31

32

33

34

35

36

37

38

39

40

41

42

43

44

45

46

47

48

49

50

51

52

53

54

55

56

57

58

59

60

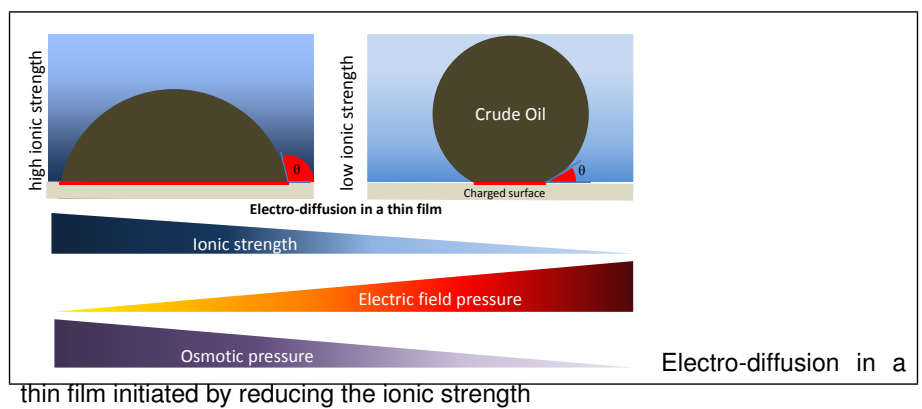

